\title{
Designing silicon-core fiber tapers for efficient supercontinuum generation in the greenhouse gas absorption region
}

\author{
J. CAmpling ${ }^{1}, *$, P. Horak ${ }^{1}$, And A. C. PeAcock ${ }^{1}$ \\ ${ }^{1}$ Optoelectronics Research Centre, University of Southampton, Southampton SO17 1BJ, UK \\ * Corresponding author: j.campling@soton.ac.uk \\ Compiled May 17, 2020
}

\begin{abstract}
We propose a tapered silicon-core optical fiber design for extending the long-wavelength edge of supercontinuum generation to obtain a high spectral density source across the $3-4.5 \mu \mathrm{m}$ regime. The taper works by generating sufficient spectral broadening of the driving laser pulse to produce a series of pumps for nondegenerate four-wave-mixing, and then opening up new phase-matching conditions to transfer the power from these pumps to a target region of mid-infrared wavelengths. We show, by simulation, that this taper design works effectively when pumped with a conventional $2.1 \mu \mathrm{m}$ femtosecond fiber-laser, significantly improving the spectral coverage obtained with a fixed diameter fiber. Thus, these tapered silicon-core fibers offer a potential platform for an efficient all-fiber spectroscopy solution to measure greenhouse gases. () 2020 Optical Society of America
\end{abstract}

http://dx.doi.org/10.1364/ao.XX.XXXXXX

\section{INTRODUCTION}

Supercontinuum (SC) generation is of great interest for its myriad of applications, such as interferometry, optical coherence tomography, confocal microscopy and spectroscopy [1-4]. Most commonly, SC spectra are generated using a high power pulsed laser to excite nonlinear processes within an optical waveguide, either planar or fiber-based [5]. The material of the waveguide is chosen to have low losses and a high nonlinearity in the spectral region of interest. The waveguide dimensions are then set to engineer the dispersion, often to position the zero-dispersion wavelength (ZDW) close to the pump in order to maximize phase-matching to the newly generated wavelengths. Usually the waveguide has a fixed core size along its length, but sometimes it is tapered such that the dimensions vary longitudinally. There are several benefits of tapering, from enhancing the coupling into and out of the waveguide to parabolic pulse-shaping [6]. It can also be used to enhance SC generation, as the varying core results in a constantly changing ZDW, helping to increase the number of phase-matched processes $[7,8]$.
Many of the early investigations focused on SC spanning near-infrared to visible wavelengths, owing to the low losses of silica glass fibers in this region [9]. However, over the past decade, attention has turned to SC generation in the midinfrared (mid-IR) spectral region, where the potential applications extend to environmental sensing and healthcare [10-12]. This shift has been partially motivated by the excellent progress in producing low loss semiconductor waveguides with extended long wavelength transparency. In particular, there is currently great interest in generating efficient and flat SC covering the 3 $-5 \mu \mathrm{m}$ region for sensing of greenhouse gases [13]. This work has been aided by the increasing availability of high power fiber-based pump lasers that operate around $2 \mu \mathrm{m}$ [14]. Although most of the work conducted in this area to date has made use of well-established silicon-based (silicon-on-insulator [15] and silicon nitride [13]) planar waveguides, more recently there have been some promising results obtained in the siliconcore fiber (SCF) platform, which has the advantage of being more directly compatible with the fiber pump sources [16, 17]. Moreover, the SCFs can be tapered using a straightforward fiber post-processing procedure to precisely control the core dimensions along the entire length.

In this paper, we investigate the role of the taper profile to obtain flat and efficient SC in a SCF that extends across the $3-4.5 \mu \mathrm{m}$ region by optimizing the four-wave mixing (FWM) phase-matching conditions. A key goal is to achieve output SC spectra at practical power levels using standard commercially available fiber laser pump sources. The taper is designed to have large input and output diameters to maximize the coupling efficiencies, with a waist such that the ZDW is close to the pump in order to enhance the efficiency of the nonlinear processes. However, in contrast to conventional tapers, the design is asymmetric such that the up-tapered output section can be carefully engineered to access new phase-matching conditions for FWM, thus extending the long wavelength substantially beyond what could be achieved with a straight waveguide. We demonstrate this with four designs, boosting the average power of the longwavelength edge of the SC reached with increasing input peak powers. 


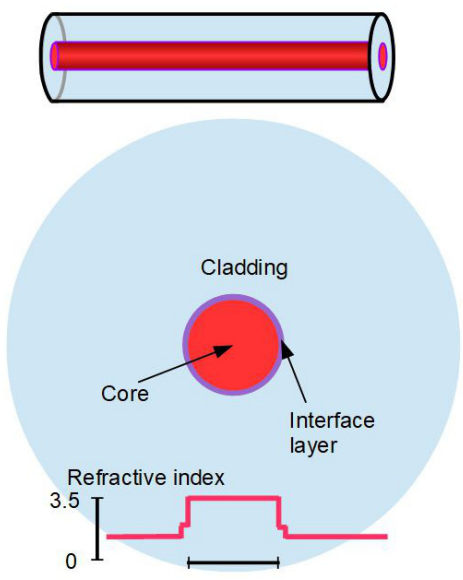

Fig. 1. Cross section of a silicon-core fiber showing core, cladding and interface layers.

\section{SILICON-CORE FIBERS AND TAPER DESIGN}

\section{A. Fabrication}

Silicon-core fibers (SCFs) are a versatile platform for nonlinear optics as they are relatively straightforward to make, have high nonlinear coefficients, and comparatively low optical losses [18]. Furthermore, their size and circular geometry makes them compatible with fiber lasers as well as standard single-mode fibers. Most commonly, SCFs are produced by the molten core drawing technique [19], which is an adaptation of the traditional fiber drawing method. The fiber preform is made by sleeving a silicon rod inside a silica capillary, which has been coated with an interface layer of calcium oxide to prevent diffusion of oxygen from the silica to the silicon [20]. The interface layer also reduces stresses on the materials during the drawing process. A schematic of a SCF is shown in Figure 1. As the SCFs are clad in silica, they are robust, easy to handle, and can be subsequently heated and stretched using conventional fiber tapering methods to precisely control the core dimensions along the length.

\section{B. Taper Design}

In this paper we look at four taper designs, each of which is optimized for a different pump power. The primary goal is to search for designs that maximize the spectral width of the supercontinuum, whilst retaining a high spectral density in the mid-IR region of interest. The tapers are divided into three sections, a down-taper (1), up-taper (2) and constant-diameter end facet (3), as shown in Fig. 2. Section (1) has a large input facet in order to couple the light into the fiber with minimal loss, which tapers down towards the waist diameter. As the light propagates in this section, it undergoes initial spectral broadening via selfphase modulation (SPM). At the waist diameter, the nonlinearity reaches its maximum and part of the spectrum crosses the zerodispersion wavelength (ZDW) to trigger soliton fission, which speeds up this broadening. Section (2) is an up-taper, where the increasing diameter opens up new phase-matching conditions for non-degenerate FWM, which acts to improve the transfer of power to increasingly longer wavelengths. This up-taper culminates in Section (3), the end-facet, where the diameter is optimized for phase-matching to the target wavelength range for the specific pump power. The larger the diameter, the longer the wavelengths that can be phase-matched to in this manner. For each design, the total length of the tapered structure and
(2)

(3)

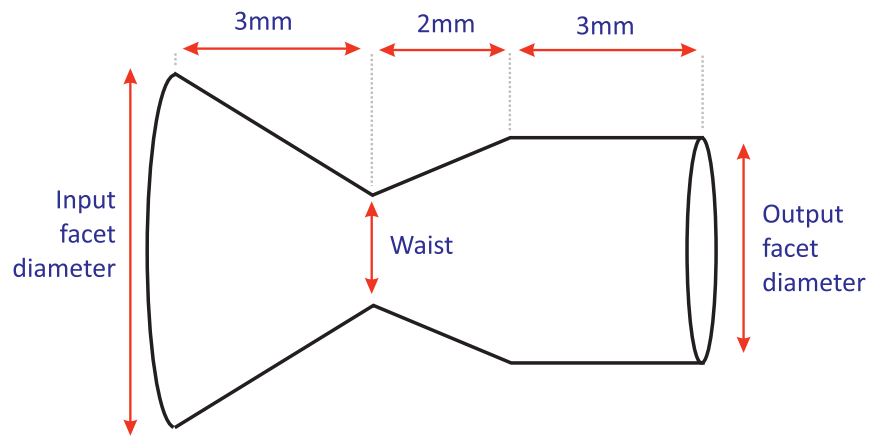

Fig. 2. Schematic showing taper design: (1) down-taper, (2) up-taper, (3) constant diameter end facet.

the three sections are fixed, but the diameters of the individual sections are allowed to vary.

\section{Length}

When deciding upon appropriate length scales for each section in the taper designs, the main factor we took into account was the nonlinear length, defined as $L_{\mathrm{NL}}=1 / \gamma P_{0}$, where $\gamma=n_{2} \omega_{0} / c A_{\text {eff }}$ is the nonlinear parameter, $n_{2}$ is the nonlinear refractive index, $\omega_{0}$ is the angular frequency of the pump, $c$ is the speed of light, $A_{\text {eff }}$ is the effective area of the fundamental mode and $P_{0}$ is the peak power of the pump. For mid-IR propagation, silicon-core diameters on the order of a few micrometers $\sim 1.5-4 \mu \mathrm{m}$ are desired as this ensures that the pump is positioned close to the ZDW and that the interaction with the silica cladding, which is lossy at these wavelengths, is minimized. Such diameters correspond to nonlinear parameters $\gamma$ in the range $3-22 \mathrm{~W}^{-1} \mathrm{~m}^{-1}$. The properties of the pump laser used in our investigations are modelled on typical values for a $2.1 \mu \mathrm{m}$ fiber laser, with a pulse duration of $150 \mathrm{fs}$, peak powers in the range $1-5 \mathrm{~kW}$, and a repetition rate of $100 \mathrm{MHz}$ [14]. Thus, for these values of $\gamma$ and $P_{0}$, typical nonlinear lengths are in the range $0.01-0.3 \mathrm{~mm}$, so we can assume significant nonlinear effects will occur over lengths of $\sim 1 \mathrm{~mm}$. However, the peak power does drop rapidly as the pulse propagates along the length, due to linear and nonlinear losses, and so most of our simulations show that the SPM broadening reaches a maximum at lengths $\sim 3 \mathrm{~mm}$. SCF tapers as short as $8 \mathrm{~mm}$ [21] have been demonstrated, we set this as our total length. The taper was then divided up to have a $3 \mathrm{~mm}$ down-taper (where most of the SPM occurs), 2 mm up-taper (where cascading FWM occurs) and $3 \mathrm{~mm}$ end-facet (to maximize phase-matching for FWM-based transfer of power to the target wavelength-range). This design is shown in Fig. 2.

\section{Controlling the initial broadening in the down-taper}

The fiber design starts with a down-tapered region, from the input facet to the waist, to facilitate efficient coupling of the fiber-based pump source into the narrow waist. During this transition, SPM initiates spectral broadening. Then, in the waist region, the red edge of the spectrum crosses the ZDW, triggering soliton fission which facilitates further broadening.

However, if too much power crosses the ZDW, the solitons that form in the anomalous dispersion region are very high order solutions, which are prone to break-up [5]. When soliton fission occurs, two problems arise: (1) the solitons recoil too far to- 
ward shorter wavelengths, wasting power that could otherwise be transferred to longer wavelengths. (2) The dispersive wave generation spreads to longer wavelengths too rapidly, which means that power does not build up in the target output wavelength ranges. Furthermore, spectral power does not build up in the central part of the spectrum, so there is no stable source of pumps for later transfer to the target ranges.

However, we do want a limited amount of soliton fission to provide additional spectral broadening to that produced by SPM. Specifically, the goal is to produce a reasonably broad intermediate spectrum with an evenly distributed spectral density in the down-tapered section, where the newly generated wavelengths can act as FWM pumps to extend the transfer of power to the mid-IR wavelengths in the final two sections of the taper. For the lower range of input powers considered in this study (1 $2 \mathrm{~kW}$ ), the waist diameter is set to $1700 \mathrm{~nm}$ (where the ZDW is at the input wavelength, $2.1 \mu \mathrm{m}$ ). For the increasingly higher input powers $(\geqslant 3 \mathrm{~kW})$, we find that it is necessary not only to increase the waist, but also the input facet, in order to control the initial SPM and limit the degree of subsequent soliton fission, as will be described in Section 4. Furthermore, unlike most taper designs in which the waist is held constant over some length to maximize a particular nonlinear process, our design transitions immediately into the up-tapered region to start continuously shifting the FWM phase-matching conditions to progressively longer wavelengths. The up-taper section of the design ends when the optimum diameter is reached for FWM wavelength conversion to the targeted wavelength region.

\section{E. Optimizing the end-facet diameter for phase-matching to the target}

This is the most important section of the design, as it is the point at which phase-matching to the target region is maintained. In order to determine the optimum output diameter for the different taper designs, we calculated the phase-matching conditions for combinations of pumps, signals and idlers covering the 1.5 $-5 \mu \mathrm{m}$ range for each diameter (sampling pump wavelengths at $25 \mathrm{~nm}$ intervals). We were then able to use this dataset to obtain the number of phase-matched solutions depending on where the pump, signal and idler wavelengths lie. For the final designs, only the subsets containing pump wavelengths longer than $1.85 \mu \mathrm{m}$ were considered for the non-degenerate FWM processes. Although solutions do exist with pumps at shorter wavelengths, they are not useful because they are only generated by excessive soliton fission in the waist region (see Appendix). Fig. 3 shows the positions of pairs of phase-matched pumps (short and long wavelength plotted as black and yellow points respectively) and corresponding idlers for two of the signal wavelength ranges, (a) $3.5-3.75 \mu \mathrm{m}$ and (b) $4.25-4.5 \mu \mathrm{m}$. Fig. 4 summarizes the results of this analysis for all four ranges, showing the usable bandwidth of the generated pumps. The maximum bandwidth corresponds to the point where the phasematched pumps reach the short-wavelength edge of the target spectral range, and this indicates the optimum output diameter for maximal transfer of power to the target range.

The phase-matching conditions for non-degenerate FWM are generally satisfied when the pumps are spaced roughly symmetrically around the $\mathrm{ZDW}$. This can be understood by recalling the phase-matching condition for non-degenerate FWM:

$$
\Delta \beta=\beta_{p 1}+\beta_{p 2}-\beta_{s}-\beta_{i}=0,
$$

where $\beta_{p 1}, \beta_{p 2}, \beta_{s}$ and $\beta_{i}$ are the propagation constants of the (a) $3.5-3.75 \mu \mathrm{m}$

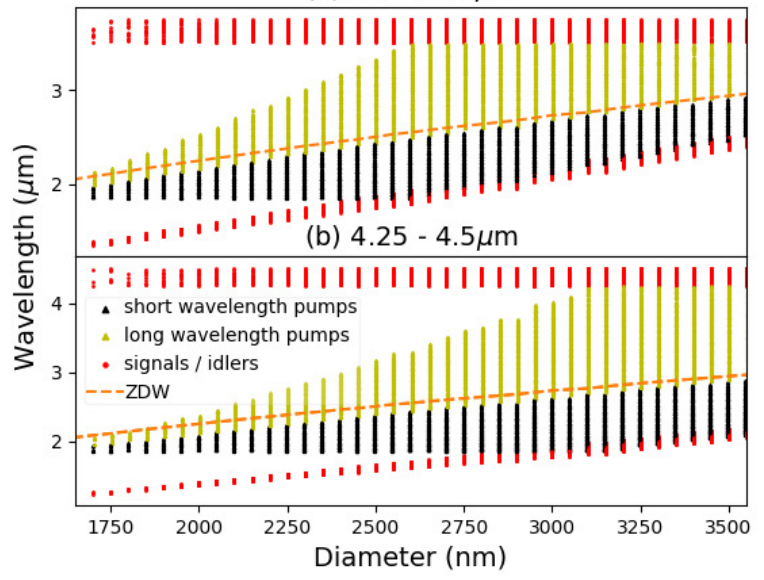

Fig. 3. Four wave mixing phase-matched solutions where pumps have $\lambda>1.85 \mu \mathrm{m}$ and signals are in the target ranges (a) $3.5-3.75 \mu \mathrm{m}$ and (b) $4.25-4.5 \mu \mathrm{m}$. Orange dashed line shows the zero-dispersion wavelength. Note how the shortwavelength pumps and long-wavelength pumps tend to cluster either side of the ZDW.

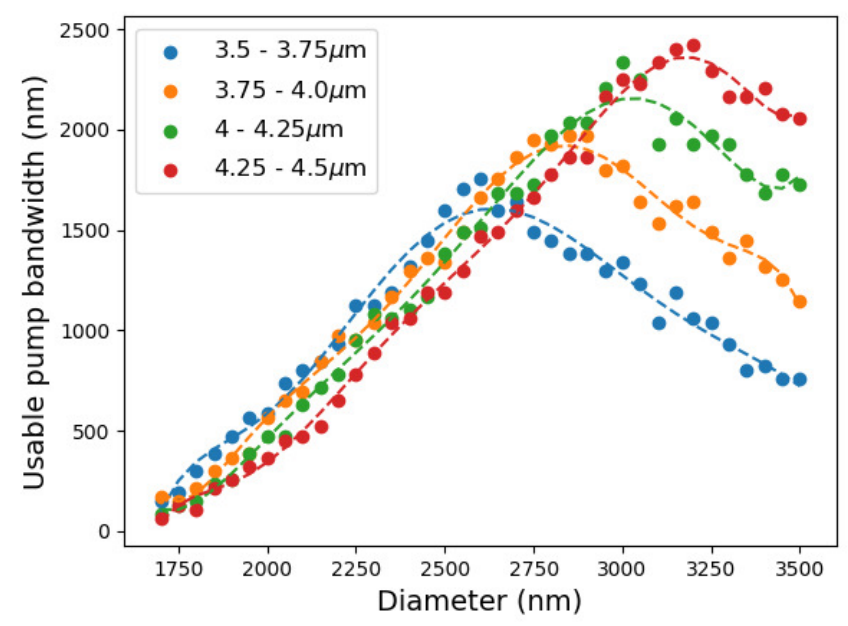

Fig. 4. Bandwidth of phase-matched solutions for nondegenerate FWM where the pumps exist at wavelengths longer than $1.85 \mu \mathrm{m}$ for target signals in the labelled wavelength ranges. The peaks correspond to the optimum output diameter for the target wavelength range.

two pumps, signal and idler beams, respectively, and the frequencies are conserved such that

$$
\omega_{p 1}+\omega_{p 2}-\omega_{s}-\omega_{i}=0
$$

By picking a reference frequency $\omega_{r}$ halfway between $\omega_{p 1}$ and $\omega_{p 2}$, we can write the propagation constant for each wavelength as a Taylor expansion:

$$
\begin{aligned}
\beta\left(\omega_{m}\right)=\beta_{0}+\beta_{1}( & \left.\omega_{m}-\omega_{r}\right)+\frac{\beta_{2}}{2 !}\left(\omega_{m}-\omega_{r}\right)^{2} \\
+ & \frac{\beta_{3}}{3 !}\left(\omega_{m}-\omega_{r}\right)^{3}+\frac{\beta_{4}}{4 !}\left(\omega_{m}-\omega_{r}\right)^{4} \ldots
\end{aligned}
$$

where $m=p 1, p 2, s, i$ (i.e. pump 1, pump 2, signal and idler respectively) and $\beta_{n}=\mathrm{d}^{n} \beta /\left.\mathrm{d} \omega^{n}\right|_{\omega=\omega_{r}}$. 
When each propagation constant is written out in this way, and substituting $\omega_{p 2}-\omega_{r}=-\left(\omega_{p 1}-\omega_{r}\right)$ and $\omega_{i}-\omega_{r}=$ $-\left(\omega_{s}-\omega_{r}\right)$ into Eq. (1), the odd-numbered $\beta_{m}$ terms vanish and we are left with only even terms:

$$
\begin{aligned}
\Delta \beta=\beta_{2}\left[\left(\omega_{p 1}-\right.\right. & \left.\left.\omega_{r}\right)^{2}+\left(\omega_{s}-\omega_{r}\right)^{2}\right] \\
& +\frac{2 \beta_{4}}{4 !}\left[\left(\omega_{p 1}-\omega_{r}\right)^{4}+\left(\omega_{s}-\omega_{r}\right)^{4}\right]+\ldots
\end{aligned}
$$

In the case that $\beta_{4}\left(\omega_{r}\right) \approx 0$, i.e., when $\beta_{3}(\omega)$ is constant, the first term in Eq. 4 is dominant, and so $\Delta \beta=0$ only when $\beta_{2}\left(\omega_{r}\right)=0$. In such a case, pairs of phase-matched pump solutions exist adjacent to the ZDW, and then at ever-increasing frequencyspacings either side of it until they reach either the lower pump limit or the target wavelength range. The maximum number of solutions will therefore exist when these limits are equallyspaced around the ZDW, i.e. the ZDW, lies halfway between them.

In the case of the silicon-core fibers considered here, the higher-order terms (as calculated by a finite-difference method) are small but not negligible. However, an investigation into the solutions which includes the $\beta_{4}$ and $\beta_{6}$ terms and various pump and signal combinations reveals that phase-matching is generally satisfied when $\beta_{2}\left(\omega_{r}\right)<0.07 \mathrm{ps}^{2} \mathrm{~m}^{-1}$, which is close enough to zero that the majority of the solutions do cluster above and below the ZDW as described above.

Indeed, Fig. 3 (which accounts for $\beta_{n}$ up to order 7) shows that the majority of the long wavelength pumps (yellow points) cluster above the ZDW (shown by the orange dashed line), and the short-wavelength ones (black points) cluster below it for the two wavelength ranges considered. These show how the ZDW increases with diameter and, as it reaches the halfway point between the lower pump limit and the lower edge of the target wavelength range, the available phase-matched solutions for FWM to occur 'fill-in' the space between these limits. This means that FWM can draw from pumps across the entire spectrum to transfer to the target wavelength range, and thus the 'density' of solutions, corresponding to the usable pump bandwidth, is maximized, as shown in Fig. 4. The position of these peaks indicates the output facet core diameter required for maximizing the output in the required range. So for maximizing power in the $3.5-3.75 \mu \mathrm{m}$ range, the diameter should be $\sim 2600 \mathrm{~nm}$ (where the ZDW is at $2.55 \mu \mathrm{m}$ and $\beta_{2}=0.38 \mathrm{ps}^{2} \mathrm{~m}^{-1}$ at $2.1 \mu \mathrm{m}$ ) whereas for the $4.25-4.5 \mu \mathrm{m}$ range, the optimum diameter is $\sim 3150 \mathrm{~nm}$ (where the ZDW is $2.8 \mu \mathrm{m}$ and $\beta_{2}=0.47 \mathrm{ps}^{2} \mathrm{~m}^{-1}$ at $2.1 \mu \mathrm{m}$ ).

\section{FIXED-DIAMETER FIBER SIMULATIONS}

In order to establish a set of useful benchmarks from which to compare the wavelength conversion achieved by the taper designs, we first had to study SC generation in a variety of silicon-core fibers with fixed-diameters. The simulations used the nonlinear Schrödinger equation, modified from [22] to include the frequency-dependence of the linear and nonlinear losses:

$$
\begin{array}{r}
\frac{\partial A(z, t)}{\partial z}=\mathrm{i} \sum_{n=2}^{7} \frac{\mathrm{i}^{n} \beta_{n}}{n !} \frac{\partial^{n} A}{\partial t^{n}}+\mathrm{i}\left(\gamma\left(\omega_{0}\right)+\mathrm{i} \gamma_{1} \frac{\partial}{\partial t}\right)|A(z, t)|^{2} A(z, t) \\
-\frac{1}{2 A_{\mathrm{eff}}} \beta_{\mathrm{TPA}}(t) *|A(z, t)|^{2} A(z, t) \\
\quad-\frac{1}{2} \alpha_{l}(t) * A(z, t)-\frac{1}{2} \sigma_{f} \cdot A(z, t)
\end{array}
$$

In Eq. (5), $\omega_{0}$ is the angular frequency of the pump, $A$ is the pulse envelope, $t$ is time, $z$ is propagation distance, $\beta_{n}$ are the dispersion coefficients at the pump, and $\gamma_{1}$ is the self-steepening term, approximated as $\gamma / \omega_{0}$. In terms of the frequency dependent nonlinear loss, this is incorporated through the two-photon absorption term $\beta_{\mathrm{TPA}}(t)$, where $\beta_{\mathrm{TPA}}(t)=\mathcal{F}^{-1}\left\{\beta_{\mathrm{TPA}}(\omega)\right\}$ (note: $*$ denotes a convolution and $\mathcal{F}$ a Fourier transform). The $\omega$-dependent TPA term is incorporated with values taken from [23]. As a result, wavelengths shorter than the pump are exposed to an increasing amount of nonlinear absorption. The frequency dependent linear loss term $\alpha_{l}(t)=\mathcal{F}^{-1}\left\{\alpha_{l}(\omega)\right\}$ is also included to account for the increased losses at wavelengths beyond $4 \mu \mathrm{m}$ associated with absorption by the silica cladding. The final term is the free-carrier absorption $\sigma_{f}=\sigma_{2}\left(1+\mathrm{i} \mu_{2}\right) N_{2}$, where $\sigma_{2}$ is the FCA cross section, $\mu_{2}$ is the free-carrier dispersion (FCD) parameter and $\mathrm{N}_{2}$ is the free carrier density, where

$$
\frac{\partial N_{2}(z, t)}{\partial t}=\frac{\beta_{\mathrm{TPA}}}{2 \hbar \omega_{0}} \frac{|A(z, t)|^{4}}{A_{\mathrm{eff}}^{2}}-\frac{N_{c}(z, t)}{\tau_{2}},
$$

$\hbar$ is the Planck constant and $\tau_{2}$ is the free-carrier lifetime.

In our simulations, we assumed single-mode propagation, which is a reasonable assumption because, as shown in [24], higher-order modes have only a minor impact on the spectral broadening in SCFs. A set of simulations were conducted for straight fibers with core diameters covering the range 1 $4 \mu \mathrm{m}$. In each case, we extracted the average power within each $0.25 \mu \mathrm{m}$ spectral window covering the entire greenhouse gas absorption region $(3-4.5 \mu \mathrm{m})$ for every propagation step. Thus, it was possible to ascertain the maximum average power transferred to each window along the propagation length. As the maximum transfer to any spectral window always occurred at a propagation length shorter than $20 \mathrm{~mm}$, this length was used for every simulation. From these simulations, we found that generating a SC extending out to $3.5 \mu \mathrm{m}$ was easily achievable in all fiber diameters with a peak power of $1 \mathrm{~kW}$, so we concentrated only on the remaining four $0.25 \mu \mathrm{m}$ ranges covering $3.5-4.5 \mu \mathrm{m}$. To access the increasingly longer mid-IR ranges, the pump power was also increased systematically, as indicated in Table 1. This table summarizes the full set of results, where for each input power, the maximum power transferred to the target wavelength range attainable with a fixed-diameter fiber is shown. Note that the final increase in power is from 3 to $5 \mathrm{~kW}$ because nonlinear absorption starts to become more significant and so the amount of power transferred to longer wavelengths drops. The corresponding spectra for these results are presented

\begin{tabular}{l|l|l|l|l}
\multicolumn{2}{c|}{ Input power } & \multicolumn{2}{c|}{ Transfer to target } & \\
$\begin{array}{l}\text { Peak } \\
(\mathbf{k W})\end{array}$ & $\begin{array}{l}\text { Average } \\
(\mathbf{m W})\end{array}$ & $\begin{array}{l}\lambda \text { Range } \\
(\mu \mathrm{m})\end{array}$ & $\begin{array}{l}\text { Maximum } \\
\text { power trans- } \\
\text { ferred (mW) }\end{array}$ & $\begin{array}{l}\text { Constant } \\
\text { diameter } \\
(\mathbf{n m})\end{array}$ \\
1 & 18 & $3.5-3.75$ & 0.022 & 1275 \\
2 & 36 & $3.75-4$ & 0.021 & 1125 \\
3 & 54 & $4-4.25$ & 0.015 & 2800 \\
5 & 90 & $4.25-4.5$ & 0.039 & 2975
\end{tabular}

Table 1. Maximum average power transferred with fixeddiameter fibers of any length to the $0.25 \mu \mathrm{m}$ target ranges with labelled input powers. 

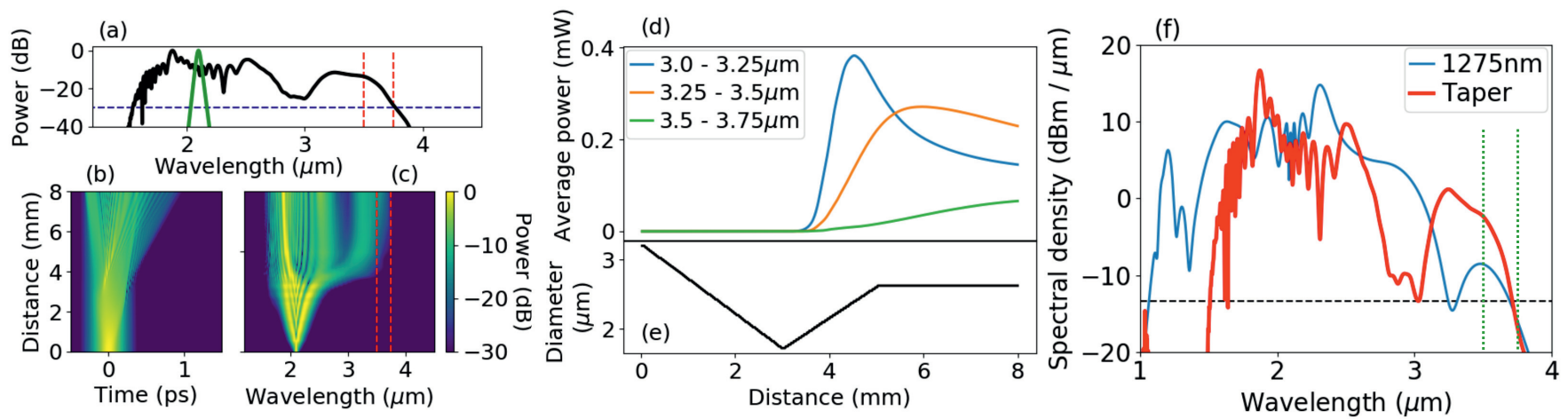

Fig. 5. $1 \mathrm{~kW}(18 \mathrm{~mW})$ pulses. (a) Output spectrum. (b) Temporal evolution. (c) Spectral evolution. (d) Evolution of average power in $0.25 \mu \mathrm{m}$ spectral ranges between 3 and $3.75 \mu \mathrm{m}$. (e) Taper profile. (f) Comparison of taper output with fixed diameter fiber (1275 nm) at length where power in the $3.5-3.75 \mu \mathrm{m}$ range is highest. Red dashed lines in (a) and (c) and green dashed lines in (f) highlight the target wavelength region.

in the following section for comparison with the results from the tapered fiber designs, where the aim of the taper was to boost the usable power in each spectral range by at least a factor of three.

\section{TAPER SIMULATION RESULTS AND DISCUSSION}

\section{A. $3.5-3.75 \mu \mathrm{m}$ target range with $1 \mathrm{~kW}$ pulses}

As explained in Section 3, with $1 \mathrm{~kW}$ peak power input pulses (18 $\mathrm{mW}$ average power), the bulk of the SC power in any fixed diameter length of fiber occurs at wavelengths shorter than $3.5 \mu \mathrm{m}$. As shown in Table 1, the maximum average power obtained in the $3.5-3.75 \mu \mathrm{m}$ window with a fixed diameter fiber is $0.022 \mathrm{~mW}$. By using our taper design (Fig. 2) with an input diameter of $3200 \mathrm{~nm}$, waist of $1700 \mathrm{~nm}$ and output facet diameter of $2625 \mathrm{~nm}$ that maximizes phase-matching to the target range, we can generate a SC with $0.066 \mathrm{~mW}$ across $3.5-3.75 \mu \mathrm{m}$, which represents a threefold improvement in power. The overall SC spans 1.2 octaves $(1.64-3.75 \mu \mathrm{m})$ with a total average power of $8.3 \mathrm{~mW}$. Fig. 5 shows the spectral output (a), as well as the evolution of the pulses in the temporal (b) and spectral (c) domains.

Fig. 5(d) shows how the average power evolves in the $0.25 \mu \mathrm{m}$ spectral windows from $3-3.75 \mu \mathrm{m}$, mapped against the taper profile below in Fig. 5(e). This evolution shows that the average power peaks in progressively longer wavelength windows as the light propagates along the length. In particular, it can be seen that the average power in the $3.25-3.5 \mu \mathrm{m}$ window peaks near the end of the up-taper region before dropping as the power in the target range $(3.5-3.75 \mu \mathrm{m})$ rises in the final straight section of the taper, reaching a maximum at the end of the section. This is evidence for FWM-based transfer of power from the shorter to the longer wavelength range along the end facet.

Finally, Fig. 5(f) shows a spectral density plot obtained using the tapered structure, compared with the optimized output from the fixed-diameter fiber $(1275 \mathrm{~nm})$ listed in Table 1 . This gives a clear indication that the tapered design can be used to improve the transfer of power to a specific spectral window.

\section{B. $3.75-4 \mu \mathrm{m}$ target range with $2 \mathrm{~kW}$ pulses}

For input pulses with a $2 \mathrm{~kW}$ peak power $(36 \mathrm{~mW}$ average power), the SC in a single diameter extends only to $\sim 4 \mu \mathrm{m}$ at the $-30 \mathrm{~dB}$ level. The maximum power transferred to the $3.75-$ $4 \mu \mathrm{m}$ window with any length of a fixed diameter is $0.021 \mathrm{~mW}$. The output facet diameter was set to $2800 \mathrm{~nm}$ to maximize phasematching to this target range. As the input power is higher, a much larger input facet diameter $(4475 \mathrm{~nm})$ is required to slow down the rate of SPM-induced spectral broadening, limiting the amount of the spectrum crossing the ZDW in the waist region, which is kept at $1700 \mathrm{~nm}$, as shown in Fig. 6(e). The
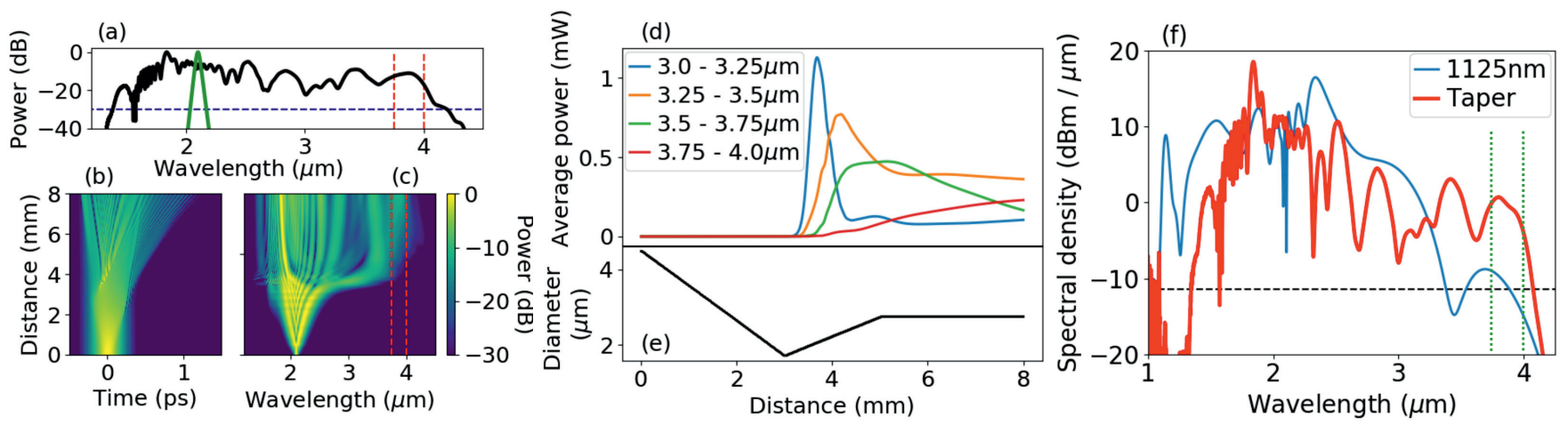

Fig. 6. $2 \mathrm{~kW}(36 \mathrm{~mW})$ pulses. (a) Output spectrum. (b) Temporal evolution. (c) Spectral evolution. (d) Evolution of average power in $0.25 \mu \mathrm{m}$ spectral ranges between 3 and $4 \mu \mathrm{m}$. (e) Taper profile. (f) Comparison of taper output with fixed diameter fiber (1125 nm) at length where power in the $3.75-4 \mu \mathrm{m}$ range is highest. Red dashed lines in (a) and (c) and green dashed lines in (f) highlight the target wavelength region. 

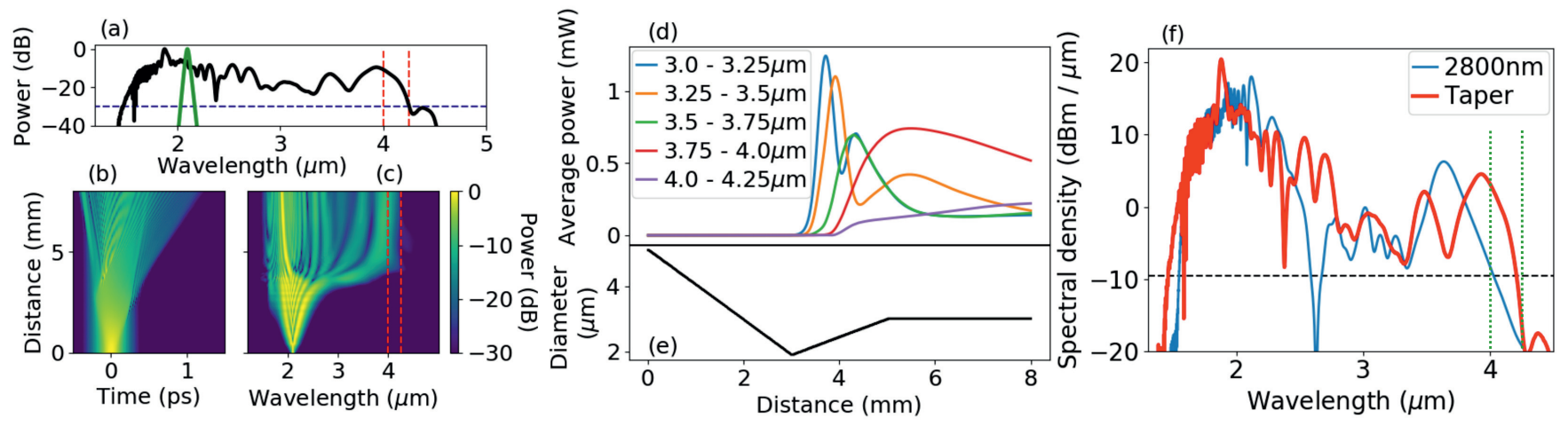

Fig. 7. $3 \mathrm{~kW}(54 \mathrm{~mW})$ pulses. (a) Output spectrum. (b) Temporal evolution. (c) Spectral evolution. (d) Evolution of average power in $0.25 \mu \mathrm{m}$ spectral ranges between 3 and $4.25 \mu \mathrm{m}$. (e) Taper profile. (f) Comparison of taper output with fixed diameter fiber (2800 nm) at length where power in the $4-4.25 \mu \mathrm{m}$ range is highest. Red dashed lines in (a) and (c) and green dashed lines in (f) highlight the target wavelength region.

downward transition from a larger input facet ensures that the spectral broadening is contained so that there is enough power in the $1.85-3.75 \mu \mathrm{m}$ region to act as pump sources during the up-taper transition, ultimately allowing for efficient FWM conversion $(0.23 \mathrm{~mW})$ to the target region, as shown in Fig. 6(a). This is an order of magnitude improvement in the useable output power compared to what was obtained in the fixed-diameter case $(0.021 \mathrm{~mW})$. The power in the $3.5-3.75 \mu \mathrm{m}$ window is also boosted to $0.166 \mathrm{~mW}$, and the average power across the entire $3-$ $4 \mu \mathrm{m}$ region is $0.8 \mathrm{~mW}$, which is sufficient power for use in practical spectroscopy applications. It is also considerably higher than the $0.3 \mathrm{~mW}$ level which has been produced with other systems that are pumped with $<10 \mathrm{~kW}$, such as aluminium nitride photonic chip waveguides [25]. The overall SC spans 1.4 octaves, from $1.6-4.18 \mu \mathrm{m}$, with a total average power of $11.8 \mathrm{~mW}$.

Fig. 6(d) shows how the average power in the four $0.25 \mu \mathrm{m}$ bands covering the $3-4 \mu \mathrm{m}$ range evolve along the taper profile, shown in Fig. 6(e). It is clear that the first three bands are largely generated in the up-taper section, before being transferred to the target range in the end facet.

Fig. 6(f) compares the spectral output from the fixed-diameter fiber $(1125 \mathrm{~nm})$ with maximum output in the $3.75-4 \mu \mathrm{m}$ range with the optimized output from the taper. Again, it is clear that the taper design introduces new phase-matching conditions that are not otherwise available, allowing for effective redistribution of the spectrum to the target region.

\section{C. $4-4.25 \mu \mathrm{m}$ target range with $3 \mathrm{~kW}$ pulses}

For input pulses with a $3 \mathrm{~kW}$ peak power $(54 \mathrm{~mW}$ average power), the power transfer to the $4-4.25 \mu \mathrm{m}$ region attainable with a fixed diameter fiber is limited to $0.015 \mathrm{~mW}$, so this is the range we wanted to boost. However, with this level of input power, we found that increasing the input facet diameter alone was not sufficient to limit the amount of spectral power crossing the ZDW before the taper waist was reached, and thus we also increased the waist diameter to $1875 \mathrm{~nm}$ in order to push the ZDW further from the pump. With the input facet diameter set to $5100 \mathrm{~nm}$, and the larger waist, this resulted in controlled broadening until the pump entered the up-tapered region where the FWM processes dominate. The output facet was increased to $3000 \mathrm{~nm}$ to optimize the phase-matching conditions for transfer to the $4-4.25 \mu \mathrm{m}$ range. With this design, the average power transferred to the target range was boosted to $0.221 \mathrm{~mW}$, as shown in Figs. $7(\mathrm{a}-\mathrm{c})$. Again, this represents an order-of-magnitude improvement in average power over the fixed-diameter result. The power evolution and spectral comparison with the fixed-diameter result are shown in Figs. 7(d-f).
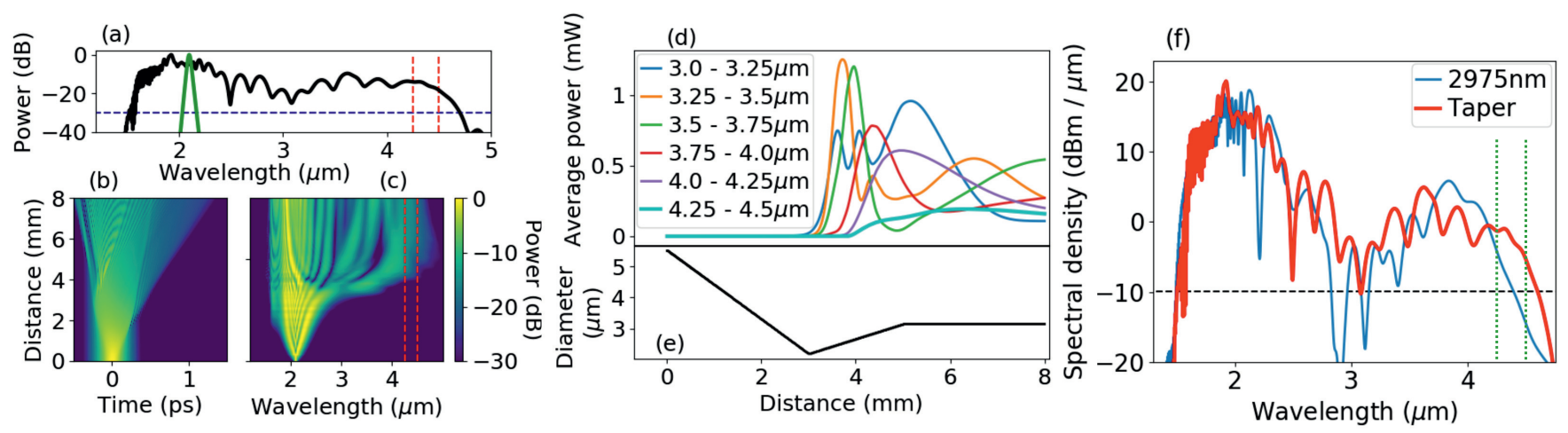

Fig. 8. $5 \mathrm{~kW}(90 \mathrm{~mW})$ pulses. (a) Output spectrum. (b) Temporal evolution. (c) Spectral evolution. (d) Evolution of average power in $0.25 \mu \mathrm{m}$ spectral ranges between 3 and $4.5 \mu \mathrm{m}$. (e) Taper profile. (f) Comparison of taper output with fixed diameter fiber (2975 nm) at length where power in the $4.25-4.5 \mu \mathrm{m}$ range is highest. Red dashed lines in (a) and (c) and green dashed lines in (f) highlight the target wavelength region. 


\section{D. $4.25-4.5 \mu \mathrm{m}$ target range with $5 \mathrm{~kW}$ pulses}

The maximum output average power achievable in the 4.25 $4.5 \mu \mathrm{m}$ spectral window with a fixed diameter fiber is $0.039 \mathrm{~mW}$, which was obtained in a fiber with a diameter of $2975 \mathrm{~nm}$ and a peak pump power of $5 \mathrm{~kW}$. To boost this range, we increased the taper input diameter to $5500 \mathrm{~nm}$ and the waist to $2175 \mathrm{~nm}$, and set the output facet diameter to $3150 \mathrm{~nm}$ to optimize phasematching to the target region. The pulse evolution is shown in Fig. 8(a-c), from which we find that the output average power in the target region is boosted to $0.159 \mathrm{~mW}$, a fourfold improvement over the fixed-diameter case. The overall SC extends from 1.6 $-4.7 \mu \mathrm{m}$ (1.5 octaves), as shown in Fig. 8(a). This would be useful for greenhouse gas spectroscopy, as there is good spectral density across the entire $3-4.5 \mu \mathrm{m}$ range, with $>0.1 \mathrm{~mW}$ of average power per $0.25 \mu \mathrm{m}$ at the output, as shown in Fig. 8(d).

\section{E. Discussion}

Table 2 summarizes the results in this section. It shows that with a low input peak power $(1 \mathrm{~kW})$, the taper design offers a threefold improvement in the average power conversion to the target wavelength range. With $2-3 \mathrm{~kW}$, the improvement is more significant, up to an order-of-magnitude. The wavelength conversion with a $5 \mathrm{~kW}$ input is more modest, as at these power levels nonlinear losses have a clamping effect, but nonetheless the average power transferred to the target region is still a fourfold improvement over that achievable with a fixed diameter fiber. However, it is clear that for higher input powers, the taper design offers limited scope for improvement. Thus we expect that our designs will be much more useful for integration with lower power sources, where the efficiency of power transfer to longer wavelengths can be significantly improved. Hence the use of a taper is ideal for a use-case where laser power is limited.

It is important to note that the principles upon which the taper design is based are general and do not depend on the particular set of wavelengths which we have studied here. We looked at these to give a concrete use-case (greenhouse-gas spectroscopy), but the design could be easily adapted for other materials, waveguide geometries and wavelength regions.

\section{CONCLUSION}

In this paper we have looked at four taper designs, each optimized according to input power in order to boost the output power on the long-wavelength side of the supercontinuum generation to a level that is not attainable with a fixed diameter fiber. The simulations show that the principle of using a tapered waveguide to extend the long-wavelength edge of a SC is sound.
In particular, we have shown that silicon-core tapered fibers offer a solution which could generate a usable amount of output power (of around $0.2 \mathrm{~mW}$ per $0.25 \mu \mathrm{m}$ spectral band) in the greenhouse-gas absorption region of the mid-IR $(3-4 \mu \mathrm{m})$, with modest input powers and pulse widths that are readily available in a portable set-up.

Although there have been various demonstrations of multiwatt average output powers in this absorption window, they typically require very high peak powers of tens of kilowatts and sub- 100 fs pulses. Thus our design is unique in that we can generate practical levels of mid-IR light using conventional fiber-laser sources with $\ll 10 \mathrm{~kW}$ peak power, offering a much more efficient solution. Furthermore, the silicon-core fiber-based design offers straightforward integration with standard glass fiber components, as they can spliced directly together, allowing for an all-fiber solution to mid-IR spectroscopy.

\section{APPENDIX: POORLY OPTIMIZED TAPER}

To demonstrate the problems caused by significant spectral broadening in the input section, the simulation results presented in Fig. 9 show propagation through a taper with the same waist and output facet as the optimized taper for $2 \mathrm{~kW}$ pulses, but with an input facet reduced to $3000 \mathrm{~nm}$.

In Fig. 9(c), the spectral evolution plot shows spectral broadening, due to soliton fission, extending down to $1.2 \mu \mathrm{m}$. This happens shortly after SPM broadens the spectrum past the $1.85 \mu \mathrm{m}$ point shown as a dashed red line. As a result, the long-wavelength side of the spectrum also broadens too quickly, and the spectrum breaks up before FWM can transfer sufficient power to the target region. The output spectrum actually extends beyond the target region, but the power is not contained and thus the maximum level reached in the $3.75-4 \mu \mathrm{m}$ range is $0.08 \mathrm{~mW}$. Furthermore, this occurs at a relatively short propagation length of $6 \mathrm{~mm}$.

Fig. 9(d) shows how the power evolves within $0.25 \mu \mathrm{m}$ ranges between the $3-4 \mu \mathrm{m}$ region along the taper profile in Fig. 9(e). It shows that the two shortest wavelength ranges peak at the same length, and then the longest wavelength range reaches a lower maximum level than in Fig. 6. Thus, the cascading power transfer to the target region does not happen very efficiently.

We found that, as a general rule, if too much spectral broadening occurs at wavelengths $<1.85 \mu \mathrm{m}$, then there is insufficient power contained in the central part of the spectrum. Therefore, FWM is not able to transfer significant power to the longer wavelength target region. So our designs attempt to contain the initial broadening by increasing the input facet with increasing input power. As such, when setting the output diameter to optimize

\begin{tabular}{|c|c|c|c|c|c|c|c|}
\hline \multicolumn{2}{|c|}{ Input power } & \multicolumn{3}{|c|}{ Taper diameters (nm) } & \multicolumn{3}{|c|}{ Transfer to target } \\
\hline $\begin{array}{l}\text { Peak } \\
(\mathrm{kW})\end{array}$ & $\begin{array}{l}\text { Avg. } \\
\text { (mW) }\end{array}$ & Input & Waist & Output & $\begin{array}{l}\text { Wavelength } \\
\text { range }(\mu \mathrm{m})\end{array}$ & $\begin{array}{l}\text { Power } \\
\text { transfer }(\mathrm{mW})\end{array}$ & Boost factor \\
\hline 1 & 18 & 3200 & 1700 & 2625 & $3.5-3.75$ & 0.066 & 3 \\
\hline 2 & 36 & 4475 & 1700 & 2800 & $3.75-4$ & 0.23 & 11 \\
\hline 3 & 54 & 5100 & 1875 & 3000 & $4-4.25$ & 0.221 & 14.7 \\
\hline 5 & 90 & 5500 & 2175 & 3150 & $4.25-4.5$ & 0.159 & 4 \\
\hline
\end{tabular}

Table 2. Summary of taper parameters used for each peak input power and the average power transferred to the target wavelength range in each case. The boost factor is the improvement in power transferred to the target range over that obtained with a fixed diameter length, as presented in Table 1. 


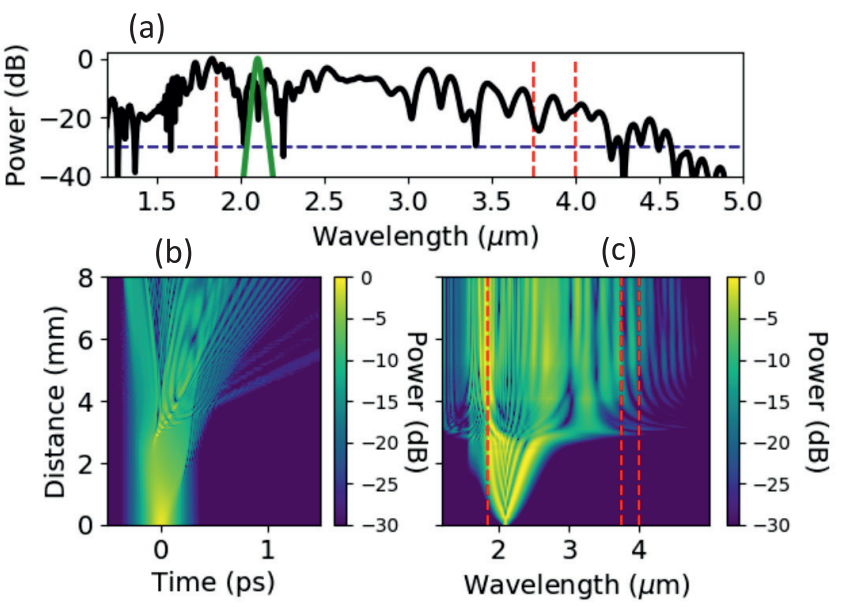

(d)
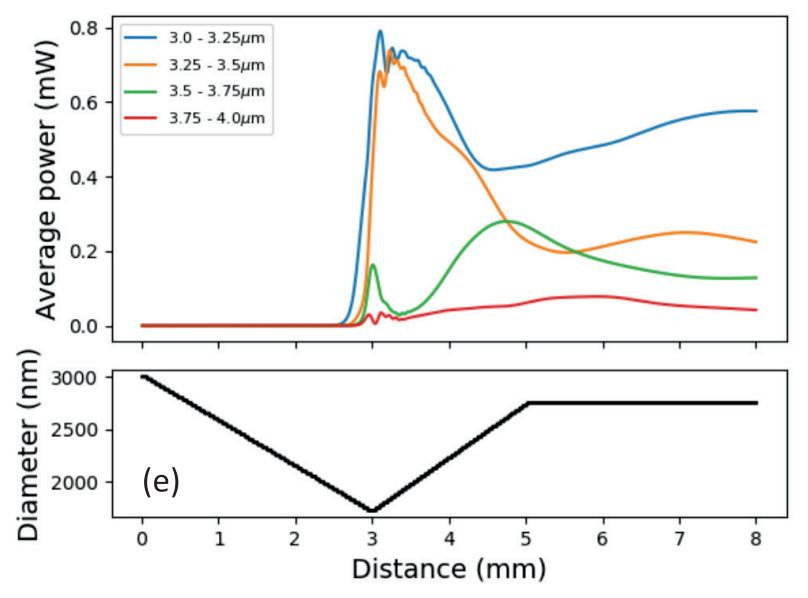

Fig. 9. $2 \mathrm{~kW}$ pulses in a taper with an input facet diameter that is smaller than optimal. (a) Output spectrum. (b) Temporal evolution. (c) Spectral evolution. (d) Evolution of average power in $0.25 \mu \mathrm{m}$ bands covering $3-4 \mu \mathrm{m}$. (e) Taper profile.

the phase-matching conditions, we only considered pumps with wavelengths $>1.85 \mu \mathrm{m}$.

\section{Funding information. EPSRC (EP/P000940/1).}

Disclosures. The authors declare no conflicts of interest.

\section{REFERENCES}

1. I. Hartl, X. D. Li, C. Chudoba, R. K. Ghanta, T. H. Ko, J. G. Fujimoto, J. K. Ranka, and R. S. Windeler, "Ultrahigh-resolution optical coherence tomography using continuum generation in an air-silica microstructure optical fiber," Opt. Lett. 26, 608-610 (2001).

2. R. K. W. Lau, M. R. E. Lamont, A. G. Griffith, Y. Okawachi, M. L., and A. L. Gaeta, "Octave-spanning mid-infrared supercontinuum generation in silicon nanowaveguides," Opt. Lett. 39, 4518-4521 (2014).

3. C. F. Kaminski, R. S. Watt, A. D. Elder, J. H. Frank, and J. Hult, "Supercontinuum radiation for applications in chemical sensing and microscopy," Appl. Phys. B 92, 367-378 (2008).

4. T. Töpfer, K. P. Petrov, Y. Mine, D. Jundt, R. F. Curl, and F. K. Tittel, "Room-temperature mid-infrared laser sensor for trace gas detection," Appl. Opt. 36, 8042-8049 (1997).

5. J. M. Dudley, G. Genty, and S. Coen, "Supercontinuum generation in photonic crystal fiber," Rev. Mod. Phys. 78, 1135-1184 (2006).

6. C. Finot, B. Barviau, G. Millot, A. Guryanov, A. Sysoliatin, and S. Wabnitz, "Parabolic pulse generation with active or passive dispersion decreasing optical fibers," Opt. Express 15, 15824-15835 (2007).
7. G. Genty, "Supercontinuum generation in dispersion-varying fibers," in Supercontinuum generation in optical fibers, J. M. Dudley and J. R. Taylor, eds. (Cambridge University Press, 2010), chap. 12, pp. 285 305.

8. T. S. Saini, T. H. Tuan, T. Suzuki, and Y. Ohishi, "Coherent mid-ir supercontinuum generation using tapered chalcogenide step-index optical fiber: experiment and modelling," Sci. Rep. 10, 1-9 (2020).

9. R. Kitamura, L. Pilon, and M. Jonasz, "Optical constants of silica glass from extreme ultraviolet to far infrared at near room temperature," App. Opt. 46, 8118-8133 (2007).

10. I. Kubat, C. Agger, P. Moselund, and O. Bang, "Mid-infrared supercontinuum generation to $4.5 \mu \mathrm{m}$ in uniform and tapered zblan step-index fibers by direct pumping at 1064 or $1550 \mathrm{~nm}$," J. Opt. Soc. Am. B 30 (2013).

11. A. B. Seddon, "A prospective for new mid-infrared medical endoscopy using chalcogenide glasses," Int. J. Appl. Glass Sci. 2, 177-191 (2011).

12. T. S. Saini, H. P. T. Nguyen, X. Luo, T. H. Tuan, T. Suzuki, and Y. Ohishi, "Broadband high-power mid-ir supercontinuum generation in tapered chalcogenide step-index optical fiber," OSA Continuum 2, 1652-1666 (2019).

13. D. Grassani, E. Tagkoudi, H. Guo, C. Herkommer, F. Yang, T. J. Kippenberg, and C.-S. Brès, "Mid infrared gas spectroscopy using efficient fiber laser driven photonic chip-based supercontinuum," Nat. Commun. 10, 1553 (2019).

14. C. W. Rudy, M. J. Digonnet, and R. L. Byer, "Advances in $2-\mu \mathrm{m}$ tmdoped mode-locked fiber lasers," Opt. Fiber Technol. 20, 642-649 (2014).

15. H. Saghaei and V. Van, "Broadband mid-infrared supercontinuum generation in dispersion-engineered silicon-on-insulator waveguide," J. Opt. Soc. Am. B 36, A193-A202 (2019).

16. H. Ren, L. Shen, A. Runge, T. Hawkins, J. Ballato, U. Gibson, and A. Peacock, "Low-loss silicon core fibre platform for mid-infrared nonlinear photonics," Light. Sci. \& Appl. 8, 105 (2019).

17. M. Huang, H. Ren, O. Aktas, L. Shen, J. Wang, T. Hawkins, J. Ballato, U. Gibson, and A. Peacock, "Fiber integrated wavelength converter based on a silicon core fiber with a nano-spike coupler," IEEE Photonics Technol. Lett. 31, 1561-1564 (2019).

18. A. C. Peacock, U. J. Gibson, and J. Ballato, "Silicon optical fibres past, present, and future," Adv. Physics: X 1, 114-127 (2016).

19. J. Ballato, T. Hawkins, P. Foy, R. Stolen, B. Kokuoz, M. Ellison, C. McMillen, J. Reppert, A. M. Rao, M. Daw, S. Sharma, R. Shori, O. Stafsudd, R. R. Rice, and D. R. Powers, "Silicon optical fiber," Opt. Express 16, 18675-18683 (2008).

20. E. F. Nordstrand, A. N. Dibbs, A. J. Eraker, and U. J. Gibson, "Alkaline oxide interface modifiers for silicon fiber production," Opt. Mater. Express 3, 651-657 (2013).

21. H. Ren, O. Aktas, Y. Franz, A. F. Runge, T. Hawkins, J. Ballato, U. J. Gibson, and A. C. Peacock, "Tapered silicon core fibers with nanospikes for optical coupling via spliced silica fibers," Opt. Express 25, 24157-24163 (2017).

22. Q. Lin, O. J. Painter, and G. P. Agrawal, "Nonlinear optical phenomena in silicon waveguides: modeling and applications," Opt. Express 15, 16604-16644 (2007).

23. N. K. Hon, R. Soref, and B. Jalali, "The third-order nonlinear optical coefficients of si, ge, and si-ge in the midwave and longwave infrared," J. Appl. Phys. 110, 011301 (2011).

24. A. C. Peacock, P. Mehta, P. Horak, and N. Healy, "Nonlinear pulse dynamics in multimode silicon core optical fibers," Opt. Lett. 37, 33513353 (2012).

25. D. D. Hickstein, H. Jung, D. R. Carlson, A. Lind, I. Coddington, K. Srinivasan, G. G. Ycas, D. C. Cole, A. Kowligy, C. Fredrick et al., "Ultrabroadband supercontinuum generation and frequency-comb stabilization using on-chip waveguides with both cubic and quadratic nonlinearities," Phys. Rev. Appl. 8, 014025 (2017). 